\title{
Downregulation of JMJD2a and LSD1 is involved in CK2 inhibition-mediated cellular senescence through the p53-SUV39h1 pathway
}

\author{
Jeong-Woo Park \& Young-Seuk Bae* \\ School of Life Sciences, BK21 FOUR KNU Creative BioResearch Group, Kyungpook National University, Daegu 41566, Korea
}

\begin{abstract}
Lysine methylation is one of the most important histone modifications that modulate chromatin structure. In the present study, the roles of the histone lysine demethylases JMJD2a and LSD1 in CK2 downregulation-mediated senescence were investigated. The ectopic expression of JMJD2a and LSD1 suppressed the induction of senescence-associated $\beta$-galactosidase activity and heterochromatin foci formation as well as the reduction of colony-forming and cell migration ability mediated by CK2 knockdown. CK2 downregulation inhibited JMJD2a and LSD1 expression by activating the mammalian target of rapamycin (mTOR)ribosomal p70 S6 kinase (p70S6K) pathway. In addition, the downregulation of JMJD2a and LSD1 was involved in activating the

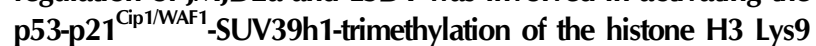
(H3K9me3) pathway in CK2-downregulated cells. Further, CK2 downregulation-mediated JMJD2a and LSD1 reduction was found to stimulate the dimethylation of Lys370 on p53 (p53K370me2) and nuclear import of SUV39h1. Therefore, this study indicated that CK2 downregulation reduces JMJD2a and LSD1 expression by activating $\mathrm{mTOR}$, resulting in $\mathrm{H} 3 \mathrm{~K} 9 \mathrm{me} 3$ induction by increasing the p53K370me2-dependent nuclear import of SUV39h1. These results suggest that CK2 is a potential therapeutic target for age-related diseases. [BMB Reports 2022; 55(2): 92-97]
\end{abstract}

\section{INTRODUCTION}

Cellular senescence is marked by an irreversible disruption of the cell cycle and is triggered by telomere attrition \& various genotoxic stressors, all of which ultimately activate DNA damage responses $(1,2)$. Senescent cells exhibit specific markers; which includes upregulated senescence-associated $\beta$-galactosidase

*Corresponding author. Tel: +82-53-950-6355; Fax: +82-53-943-6925; E-mail: ysbae@knu.ac.kr

https://doi.org/10.5483/BMBRep.2022.55.2.148

Received 22 October 2021, Revised 19 November 2021, Accepted 14 December 2021

Keywords: CK2, JMJD2a, LSD1, p53, Senescence-associated heterochromatin foci
(SA- $\beta$-gal) activity, triggering of the p53-p2 $1^{\text {Cip1/NAF1 }}$ pathway, and formation of senescence-associated heterochromatin foci (SAHF), all of which are a consequence of chromatin condensation to punctate its heterochromatic domains $(3,4)$. The development of SAHF downregulates the gene expression of proteins, cyclin A, and cyclin D1, the proteins related to cell cycle progression. SAHF include several markers, such as the trimethylation of histone protein, $\mathrm{H} 3 \mathrm{Lys} 9(\mathrm{H} 3 \mathrm{~K} 9 \mathrm{me} 3)$ and the binding of heterochromatin protein $1(\mathrm{HP} 1 \alpha-\gamma)$ to $\mathrm{H} 3 \mathrm{~K} 9 \mathrm{me} 3(5)$.

H3K9 methylation is mediated by histone lysine methyltransferases, including SUV39h1, G9a, and GLP (6, 7). By contrast, histone lysine demethylases, including the JmjC domain-containing histone demethylase (JMJD2/KDM4) and lysine-specific demethylase 1 (LSD1/KDM1a), remove methyl groups from H3K9 (8-11). The JMJD2 subfamily (JMJD2a-d) catalyzes the specific removal of di- and trimethyl groups from H3K9. LSD1 specifically demethylates H3K9 (either mono- or dimethylated). JMJD2a and JMJD2C cooperate with LSD1 to regulate protein lysine methylation (12). The overexpression of H3K9 demethylases is implicated in various cancer types, including prostate, bladder, and breast cancer (13). LSD1 can also target nonhistone proteins such as p53 and E2F1 $(14,15)$. Modulation of p53 activity is critically controlled by p53 protein stability, which in turn is based on post-translational modifications including phosphorylation, acetylation, methylation, and ubiquitination.

The mammalian target of rapamycin (mTOR), a protein kinase regulates cell growth based on factors like cell energy levels, the types of growth factors, and other nutrients (16). These anabolic processes are regulated by mTOR at molecular level via transcription, followed by protein synthesis, ribosome biogenesis, and the subsequently nutrient transport. The activation of mTOR leads to the phosphorylation of several proteins, such as the initiation factor $4 \mathrm{E}$ (elF4E)-binding proteins (4E-BPs) (which is the initiation factor for eukaryotic translation) and the p70 S6 kinase (p70S6K) on ribosome, and subsequently controls the downstream protein synthesis (17).

Our previous studies revealed that CK2 downregulation accelerated senescence via the $\mathrm{p} 53-\mathrm{p} 21^{\mathrm{Cip} 1 / \mathrm{WAF} 1}$ pathway in human cells and that p53 increased after reactive oxygen species production via the PI3K-AKT-mTOR pathway and transcription factor Nrf2 (18-21). The knockdown of the protein, KIN-10 which is the

ISSN: 1976-670X (electronic edition)

Copyright (c) 2022 by the The Korean Society for Biochemistry and Molecular Biology

(c) This is an open-access article distributed under the terms of the Creative Commons Attribution Non-Commercial License (http://creativecommons.org/licenses/by-nc/4.0) which permits unrestricted non-commercial use, distribution, and reproduction in any medium, provided the original work is properly cited. 
Caenorhabditis elegans orthologue of CK2 $\beta$ induces the expression of the biomarkers which are related to age, such as the decreased longevity and impedance of locomotion in C. elegans (22). Also, the downregulation of CK2 causes in turn upregulation of expression of the protein SUV39h1in a p53-dependent manner which in turn causes $\mathrm{H} 3 \mathrm{~K} 9 \mathrm{me} 3$ and the formation of SAHF (23). Hence, these findings indicate that $\mathrm{H} 3 \mathrm{~K} 9$ demethylases are downregulated during CK2 downregulation-mediated senescence.

In this study, the roles of proteins, JMJD2a, and LSD1 in the formation of SAHF, after the downregulation of protein CK2 $\beta$, have been investigated in HCT116 and MCF-7 cell lines. The results showed that JMJD2a and LSD1 overexpression successfully abrogated CK2 downregulation-induced senescence and that CK2 knockdown inhibited JMJD2a and LSD1 mRNA translation, thereby increasing p53 K370 methylation. This could lead to the stimulation \& triggering of the p53-SUV39h1-H3K9me3 pathway. This study is the first study to show that CK2 can regulate histone demethylases. As CK2 is associated with senescence and aging, therefore it is a prospective target for therapeutic purposes for age-based disorders.

\section{RESULTS}

Ectopic expression of JMJD2a and LSD1 suppressed the induction of SA- $\beta$-gal activity and SAHF formation as well as the reduction of colony-forming and cell migration ability mediated by CK2 knockdown

It was previously demonstrated that CK2 downregulation could induce SAHF formation and $\mathrm{H} 3 \mathrm{~K} 9 \mathrm{me} 3$ by upregulating the expression of H3K9 trimethyl transferase SUV39h1, in HCT116 and MCF-7 cells (22). To determine the role of H3K9 demethylases in CK2 knockdown-induced senescence, MCF-7 and HCT116 cells were treated with either pCMV-JMJD2a or pCMV-LSD1 in the presence of CK2 $\alpha$ siRNA. Consistent with a previous study (18), CK2 downregulation stimulated the SA- $\beta$-gal activity. However, the ectopic expression of JMJD2a and LSD1 inhibited the stimulation of the SA- $\beta$-gal activity significantly in CK2-knockdown cells (Fig. 1A). SAHF includes multiple markers of heterochromatin which is transcriptionally silent, including H3K9me3 and $\operatorname{HP} 1 \gamma(3,4)$. Therefore, SAHF formation was identified via 4',6-diamidino-2-phenylindole (DAPI) staining and immunofluorescence analysis with antibodies against H3K9me3 and HP1 $\gamma$. CK2 downregulation increased the fluorescence intensity of $\mathrm{H} 3 \mathrm{~K} 9 \mathrm{me} 3$ (red) and its co-localization with HP1 $\gamma$ (green) in DAPI-dense heterochromatin foci (blue), and the ectopic expression of JMJD2a and LSD1 suppressed the induction of SAHF formation in CK2-knockdown cells (Fig. 1B). Cell colony formation and migration abilities are critical processes in cancer tumorigenesis and metastasis (24). As senescence suppresses tumor progression (1), assays for analyzing the colony formation and the cell migration were performed, and the results showed that CK2 downregulation inhibited the colony formation and cell migration indices. However, ectopic expression of JMJD2a and LSD1 suppressed this reduction of colony formation and
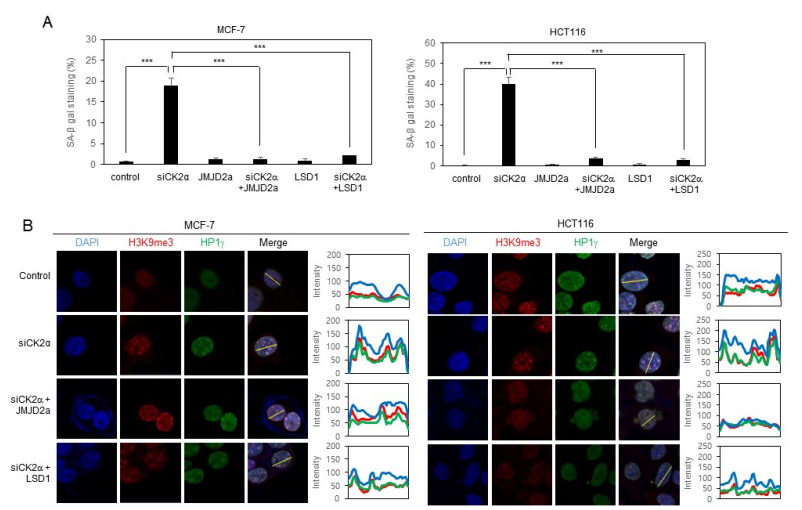

Fig. 1. Overexpression of JMJD2a and LSD1 suppresses CK2 knockdown-mediated SA- $\beta$-gal staining and SAHF formation. MCF-7 and HCT116 cells were treated with CK2 $\alpha$ siRNA in the absence or presence of pCMV-JMJD2a and pCMV-LSD1. (A) Cells were stained with 5-bromo-4-chloro-3-indolyl- $\beta$-D-galactoside, and the percentage of bluestained cells was determined. Values indicate the mean $\pm \mathrm{SEM}$. $* * * \mathrm{P}<$ 0.001 . (B) Confocal immunofluorescence images of the co-localization of chromatin foci with H3K9me3 (red) and HP1 $\gamma$ (green) in cells. DAPI staining (blue) was used to visualize DNA foci. The fluorescence intensity was quantified using Image software (right panels). Arbitrary intensity values for H3K9me3 (red), HP1 $\gamma$ (green), or DAPI (blue) are shown relative to the reference line (white) used for analysis.

cell migration abilities mediated by CK2 knockdown (Supplementary Fig. 1). These results suggest that JMJD2a and LSD1 downregulation is required for the senescence markers induced by the CK2 downregulation, including upregulated SA- $\beta$-gal staining and SAHF formation and declined colony formation and cell migration capabilities.

CK2 downregulation inhibited the expression of JMJD2a and LSD1 at the translational level by activating the mTOR-p70S6K pathway

Next, we investigated whether $\mathrm{CK} 2$ regulates H3K9 demethylase expression in cells. Treating MCF-7 and HCT116 cells using siRNA of $\mathrm{CK} 2 \alpha$ reduced the protein levels of $\mathrm{H} 3 \mathrm{~K} 9$ demethylases (JMJD2a, JMJD2c, and LSD1) compared with control cells. By contrast, treatment with pcDNA3.1-HA-CK2 $\alpha$ increased the protein levels of JMJD2a, JMJD2C, and LSD1, suggesting that CK2 may positively regulate the expression of these H3K9 demethylases (Fig. 2A). As CK2 knockdown-mediated senescence is p53-dependent (19), the effect of p53 on the expression of these demethylases was examined. Similar to cells containing wild-type p53, the protein levels of these demethylases were downregulated due to $\mathrm{CK} 2 \alpha$ knockdown and upregulated by the expression of $\mathrm{CK} 2 \alpha$ in both HCT116 p53 ${ }^{-1-}$ cells and MDA-MB-435 cells (which contained a point mutation within p53), suggesting that p53 is not involved in the CK2-mediated regulation of these demethylases (Supplementary Fig. 2). We next examined whether the decrease in the protein levels of these demethylases is correlated with a decrease at their tran- 


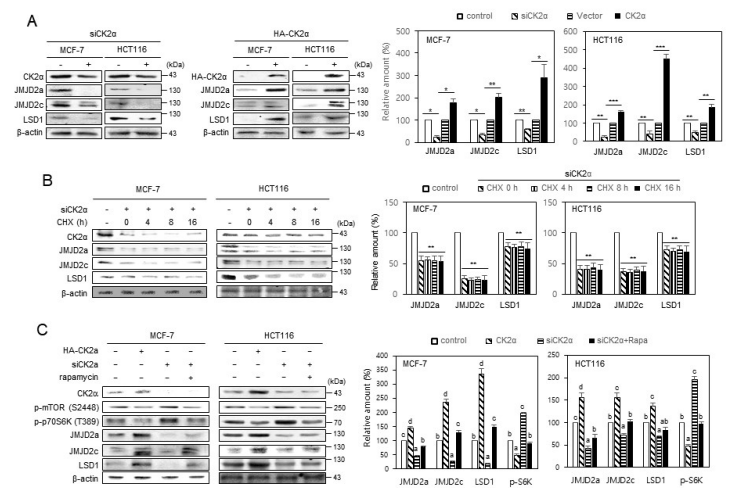

Fig. 2. CK2 downregulation decreases the expression of JMJD2a and LSD1 at the translational level by activating the mTOR-p70S6K pathway. MCF-7 and HCT116 cells were transfected with (A) CK2 $\alpha$ siRNA or pcDNA3.1-HA-CK2 $\alpha$, (B) CK2 $\alpha$ siRNA in the presence of $50 \mu \mathrm{g} / \mathrm{ml}$ of cycloheximide $(\mathrm{CHX})$ for the indicated times, or (C) CK2 $\alpha$ siRNA or pcDNA3.1-HA-CK2 $\alpha$ in the presence of $0.1 \mu \mathrm{M}$ rapamycin. After 2 days, the level of each protein was determined via immunoblot analysis using antibodies against CK2 $\alpha$, hemagglutinin (HA, for HA-CK2 $\alpha$ in Fig. 2A), JMJD2a, JMJD2c, LSD1, p-mTOR (S2448), and p70S6K (T389) (left panels). $\beta$-actin was used as a control. Graphs represent the quantification of each protein relative to $\beta$-actin (right panels). Data are shown as the mean \pm SEM. $* P<0.05$; * $* P<0.01$; ***P $<$ 0.001. Bars that do not share a common letter (a-d) are significantly different among groups at $\mathrm{P}<0.05$.

scriptional level. The mRNA levels of JMJD2a, JMJD2c, and LSD1, were not affected by either the knockdown or the increased expression of CK2 $\alpha$ which suggested that CK2 regulates the expression of these demethylases at the stage of post-transcription (Supplementary Fig. 3). Further, to investigate whether the expression of these demethylases is regulated by CK2 at the translation level, cells transfected with CK2 $\alpha$ siRNA were cultured in the presence of cycloheximide, which is the inhibitor of protein synthesis at $50 \mu \mathrm{g} / \mathrm{ml}$. The protein levels of JMJD2a, JMJD2c, and LSD1 were decreased steadily after 4-16 h of cycloheximide treatment, suggesting that CK2 knockdown reduces JMJD2a, JMJD2C, and LSD1 expression at the translational step (Fig. 2B). As the mTOR-p70S6K axis is associated with protein synthesis (17), cells were treated with CK2 $\alpha$ siRNA in the presence of rapamycin, which is the mTOR inhibitor at $100 \mathrm{nM}$. The mTOR-p70S6K pathway activation was then assessed by examining the mTOR phosphorylation and the phosphorylation of p70S6K, which is the downstream target. The results revealed that CK2 knockdown markedly increased the phospho-mTOR (S2448) and phospho-p70S6K (T389) levels. However, rapamycin treatment attenuated both the activation of mTOR-p70S6K and reduction of demethylases in CK2- knockdowned cells (Fig. 2C). Overall, these results could indicate that downregulation of CK2 decreases JMJD2a, JMJD2C, and LSD1 expression at the translational level via the activation of the mTOR-p70S6K pathway.
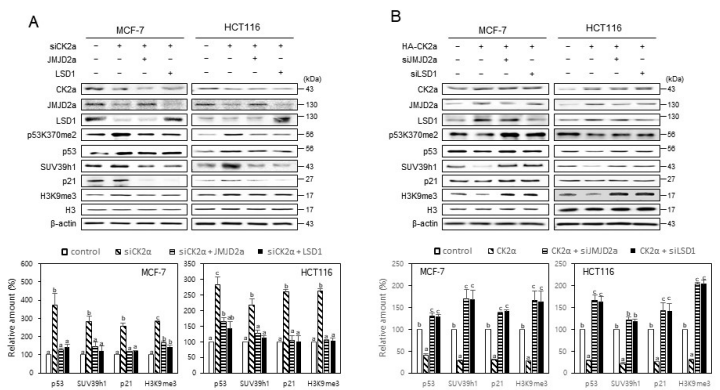

Fig. 3. CK2 downregulation-mediated reduction of JMJD2a and LSD1 activates the p53-p21 ${ }^{\text {Cip1/NAF1 }}$-SUV39h1-H3K9me3 axis. (A) Cells were treated with CK2 $\alpha$ siRNA in the absence or presence of pCMV-JMJD2a and pCMV-LSD1. (B) Cells were treated with pcDNA3.1-HA-CK2 $\alpha$ in the absence or presence of JMID2a siRNA and LSD1siRNA. Cell lysates were visualized via immunoblotting (upper panels), and graphs represent the quantification of each protein relative to $\beta$-actin (bottom panels). Data are presented as the mean \pm SEM. Bars that do not share a common letter (a-c) are significantly different among groups at $\mathrm{P}<0.05$.

\section{CK2 downregulation-mediated reduction of JMJD2a and LSD1 activated the p53-p21 ${ }^{\text {Cip1/WAF1 }}-$ SUV39h1-H3K9me3 axis} We then transfected cells with pCMV-JMJD2a or pCMV-LSD1 in the presence of $\mathrm{CK} 2 \alpha$ siRNA to investigate the roles of JMJD2a and LSD1 in H3K9me3 and SUV39h1 expression induced by CK2 downregulation. Consistent with a previous study (23), CK2 $\alpha$ knockdown caused increase in H3K9me3 levels and also increased the expression of protein, SUV39h1. However, JMJD2a and LSD1 overexpression abrogated the induction of H3K9me3 and SUV39h1 in CK2- knockdowned cells (Fig. 3A). As the p53-p21 $1^{\text {Cip1NAF1 }}$ pathway is an upstream activator of CK2 knockdown-mediated H3K9me3 and SUV39h1 expression (25), the effect of JMJD2a and LSD1 on p53 and p21 Cip1/WAF1 expression was also evaluated. The increased expression of JMJD2a and LSD1 in turn, suppressed the stimulation of p53 and p21 $1^{\text {Cip1 NAF1 }}$ in the cells where CK2 was knockdowned (Fig. $3 \mathrm{~A})$. By contrast, CK2 $\alpha$ overexpression decreased the levels of p53, p21 ${ }^{\text {Cip1/NAF1 }}$, SUV39h1, and H3K9me3. Moreover, the knockdown of JMJD2a and LSD1 attenuated the reduction of p53, p2 $1^{\text {Cip1/WAF1 }}$, SUV39h1, and H3K9me3 in CK2 $\alpha$-overexpressing cells (Fig. 3B). To examine the effect of JMJD2a and LSD1 on the transcriptional activity of p53, we performed chromatin immunoprecipitation assay with p53 antibody. The results showed that CK2 knockdown reduced binding of p53 to the promoter of $p 21^{\text {Cip } 1 / \text { WAF1 }}$, whereas the increased expression of JMJD2a and LSD1 caused suppression in the CK2 knockdown-mediated reduction of $\mathrm{p} 53$ binding to the promoter of $p 21^{\text {Cip1/WAF1 }}$ (Supplementary Fig. 4). The CK2-downregulated and JMJD2a/LSD1-upregulated cells were further treated with p53 cDNA to confirm the role of p53 in the reduction of SUV39h1, which was mediated by the ectopic expression of JMJD2a or LSD1 in CK2knockdowned cells. The induced p53 overexpression rescued the reduction of SUV39h1 that was mediated by the overex- 


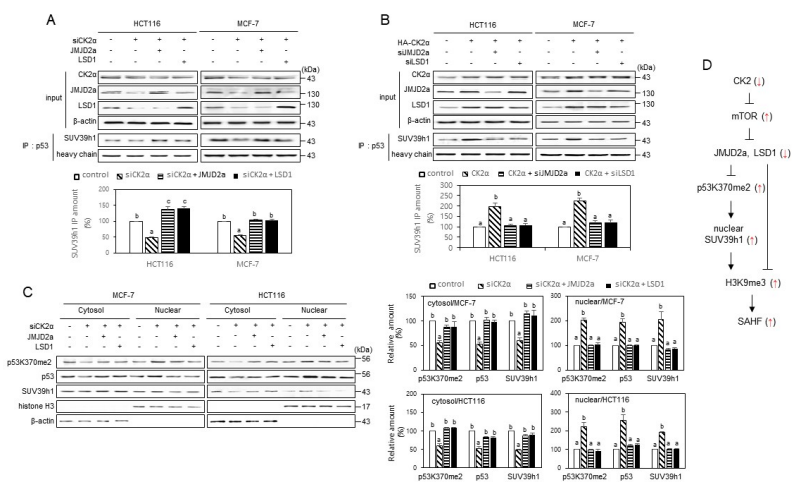

Fig. 4. CK2 downregulation-mediated JMJD2a and LSD1 reduction stimulates the dimethylation of p53 Lys370 and leads to the nuclear import of SUV39h1. (A, C) Cells were treated with CK2 $\alpha$ siRNA in the absence or presence of pCMV-JMJD2a and pCMV-LSD1. (B) Cells were treated with pcDNA3.1-HA-CK2 $\alpha$ in the absence or presence of JMJD2a siRNA and LSD1siRNA. (A, B) Cell lysates were immunoprecipitated (IP) with anti-p53 antibodies, followed by immunoblotting with anti-SUV39h1 antibodies. The cell lysate IP with IgG heavy chain served as a loading control (upper panels). Representative data from three independent experiments are shown. Graphs represent the quantification of SUV39h1 relative to the heavy chain (bottom panels). (C) The cytoplasm and nuclei were isolated from cells, and both extracts were visualized via immunoblotting. B-actin (cytoplasmic marker) and histone $\mathrm{H} 3$ (nuclear marker) were used as loading controls (left panels). Graphs represent the quantification of p53K370me2, p53, and SUV39h1 relative to subcellular markers (right panels). Values indicate the mean \pm SE. Bars that do not share a common letter (a-c) are significantly different among groups at $\mathrm{P}<0.05$. (D) Possible model illustrating the CK2 downregulation-mediated reduction of JMJD2a and LSD1 for SAHF formation.

pression of either JMJD2a or LSD1. However, p53 overexpression did not affect JMJD2a and LSD1 levels, indicating that p53 is an upstream regulator of SUV39h1 but not JMJD2a and LSD1 (Supplementary Fig. 5). Overall, these results indicated that the downregulation of JMJD2a and LSD1 in CK2-knockdowned cells activates the p53-p2 $1^{\text {Cip1/NAF1 }}$ pathway, leading to the expression of SUV39h1 and H3K9me3.

\section{CK2 downregulation-mediated JMJD2a and LSD1 reduction stimulated the dimethylation of p53 Lys370 and led to the nuclear import of SUV39h1}

We previously reported that downregulation of CK2 enzyme enhances the nuclear import and subsequent stabilization of SUV39h1 via inhibiting interaction between p53 and SUV39h1 (25). Hence, immunoprecipitation analyses were performed to determine whether JMJD2a and LSD1 regulate this interaction between p53 and SUV39h1 in CK2 $\alpha$-knockdowned cells. Compared with control cells, a lower amount of SUV39h1 was coprecipitated with p53 in CK2-downregulated cells. However, the ectopic expression of JMJD2a or LSD1 enhanced the coprecipitation of SUV39h1 with p53 in cells (Fig. 4A). By contrast, more SUV39h1 was coprecipitated with p53 in cells with CK2 $\alpha$ upregulation as compared to the control cells. Conversely, the treatment using the JMJD2a or LSD1 siRNA abrogated this effect (Fig. 4B). Overall, these results could demonstrate that the binding of p53 to SUV39h1 is decreased by reducing JMJD2a and LSD1 in CK2-knockdowned cells.

LSD1 has been reported to demethylate the dimethyl group of Lys370 on p53 (p53K370me2) $(14,15)$; however, the role of JMID2a is still unclear. Our immunoblot analysis using a p53K370me2specific antibody revealed that p53K370me2 was increased in CK2-downregulated cells and that the ectopic expression of JMJD2a or LSD1 suppressed this effect, indicating that JMJD2a and LSD1 demethylate the dimethyl group of p53K370me2 (Fig. 3A). To assess the effect of p53K370me2 on the nuclear localization of p53 and SUV39h1, the cytoplasm, and nuclei were isolated from MCF-7 and HCT116 cells. The accumulation of p53K370me2, total p53, and SUV39h1 in cells with CK2 downregulation was greater in the analyzed nuclear extracts than that of the cytosolic extracts. However, the overexpression of JMJD2a or LSD1 suppressed the nuclear transport of SUV39h1 in CK2-downregulated cells, which suggested that JMJD2a and LSD1 negatively regulate the nuclear import of SUV39h1 in cells where CK2 is downregulated (Fig. 4C). In totality, the overall results demonstrated that the reduction of JMJD2a and LSD1 as mediated by CK2 downregulation, stimulates p53K370me2, leading to the decreased interaction between p53 and SUV39h1 and the subsequent nuclear import of SUV39h1.

\section{DISCUSSION}

The downregulation of CK2 has been reported to induce the expression of several senescence markers, including activation of the p53-p2 $1^{\text {Cip } 1 / \text { WAF } 1}$ pathway and SA- $\beta$-gal staining and growth of SAHF, in human cell lines (18-23, 25). As JMJD2a and LSD1 accelerate $\mathrm{H} 3 \mathrm{~K} 9 \mathrm{me} 3$ demethylation, we examined whether these demethylases are associated with SAHF formation that is mediated by downregulation of CK2 protein. The current study demonstrated that it is the ectopic expression of either JMJD2a or LSD1 which successfully abrogated the induction of CK2 downregulation and then the senescence markers, SA- $\beta$-gal activity, p53-p21 $1^{\text {Cip } 1 / \text { NAF } 1}$ pathway activation, $\mathrm{H} 3$ K9me3, and SAHF formation as well as reduction of colony formation and cell migration abilities in HCT116 and MCF-7 cell lines. These findings indicate that the downregulation of JMJD2a and LSD1 may be required for the induction of senescence-specific phenotypes in CK2-knockdowned cells (Figs. 1 and 3; Supplementary Fig. 1). In accordance, the protein levels of JMJD2a, JMJD2C, and LSD1 were reduced by CK2 downregulation, whereas CK2 $\alpha$ overexpression increased their protein levels (Fig. 2A). Moreover, the present study indicated that CK2 downregulation decreased JMJD2a and LSD1 expression at the translational level by activating the mTOR-p70S6K pathway (Fig. 2B, C, Supplementary Fig. 3). In our previous study, CK2 downregulation was found at the transcriptional level to cause reduction in the expression of the H3K9 dimethyltransferases G9a, GLP, and SETDB1 and at the post-translational level cause the increase in the protein 
stability of the H3K9 trimethyltransferase SUV39h1 (23). Henceforth, our previous and the current study findings demonstrate that the protein CK2 regulates the expression of various histonemodifying enzymes at different steps; e.g., H3K9 dimethyltransferases (G9a, GLP, \& SETDB1) at the transcriptional level, H3K9 demethylases (JMJD2a, JMJD2C, and LSD1) at the translational level, and H3K9 trimethyltransferase (SUV39h1) at the post-translational level. The dysregulation of histone methyltransferases and demethylases is accompanied with the progression of several diseases, including cancer, and their inhibitors have already reached the first phases of clinical trials in cancer therapy $(13,26-28)$. Our findings indicated that CK2 could also be a valuable anticancer therapy target for regulating histonemodifying enzymes.

Our previous studies showed that p53 may be an important regulator in CK2 downregulation-mediated SAHF formation (23) and that the downregulation of CK2 inhibits the interaction between p53 and SUV39h1 which may enhance the import of nuclear SUV39h1 by (25). The current study showed that CK2 regulated the protein levels of JMJD2a, JMJD2C, and LSD1 in a p53-independent manner (Supplementary Fig. 2). However, the ectopic expression of JMJD2a and LSD1 suppressed the activation of p53 in CK2-downregulated cells, and the knockdown of JMJD2a and LSD1 abrogated the inactivation of p53 in CK2-upregulated cells (Fig. 3). Hence, these results indicated that JMJD2a and LSD1 are the upstream regulators of p53. In addition, histone lysine methyltransferases and demethylases have been reported to play significant roles in modifying p53 $(14,15)$. The current study demonstrated that expression of p53K370me2 was amplified in cell where CK2 was downregulated and that the ectopic expression of JMJD2a or LSD1 suppressed this increase (Fig. 3A). These results suggest that the downregulation of JMJD2a and LSD1 increases the stability of $\mathrm{p} 53$ by preventing the removal of the methyl groups from p53K370me2 in CK2-downregulated cells.

The present study demonstrated the reduced coprecipitation of SUV39h1 with p53 in CK2-downregulated cells. The ectopic expression of JMJD2a or LSD1, however, enhanced the coprecipitation of SUV39h1 with p53 in these cells. In accordance, a greater amount of SUV39h1 was coprecipitated with p53 in cells with CK2 $\alpha$ upregulation. However, the knockdown of JMJD2a or LSD1 inhibited the coprecipitation of SUV39h1 with p53 in these cells (Fig. 4A, B). In addition, the amount of SUV39h1 was higher in the nuclei than in the cytosol of cells with CK2 downregulation. The ectopic expression of JMJD2a or LSD1, however, suppressed the import of SUV39h1 into nucleus in the cells where CK2 was downregulated (Fig. 4C). In summation, the results indicate that the reduction of JMJD2a and LSD1 which in turn is mediated by downregulation of CK2 causes stimulation of the nuclear import of SUV39h1 by decreasing the interaction between p53 and SUV39h1. Previously, it was shown that the CK2 downregulation-induced dephosphorylation of S392 on p53 promoted H3K9me3 by inhibiting the interaction between p53 and SUV39h1, which resulted in the nuclear import of SUV39h1 (25). Hence, the finding of the previous and the current studies demonstrates that the protein CK2 regulates the nuclear import of SUV39h1 via two pathways: i) modulation of the p53S392 phosphorylation status by CK2 and ii) modulation of the p53K370 methylation status by JMJD2a and LSD1.

In conclusion, based on the present study results, we propose a model for the induction of $\mathrm{H} 3 \mathrm{~K} 9 \mathrm{me} 3$ during cellular senescence mediated by CK2 downregulation. The downregulation of CK2 may reduce translation of JMJD2a and LSD1 by activating the mTOR-p70S6K pathway, thereby resulting in the accumulation of p53K370me2. In addition, the dimethylation of Lys370 on p53 may prevent the binding of p53 to SUV39h1, leading to the nuclear import of SUV39h1 and subsequently increased H3K9me3 (Fig. 4D).

\section{MATERIALS AND METHODS}

Additional materials and methods are available in the Supplemental Information.

\section{SA- $\beta$-gal activity assay}

SA- $\beta$-gal activity was measured as described in a previous study (18).

\section{Immunoblot and immunofluorescence analyses}

Immunoblot analysis was performed as described in a previous study (19). Immunofluorescence analysis was performed using the anti-H3K9me3 (1:100) and HP1 $\gamma(1: 100)$ antibodies as described previously (23). DAPI was used to counterstain nuclei, and fluorescence signals were detected using Carl Zeiss Axioplan 2 microscope (Carl Zeiss, Jena, Germany). Fluorescence images were analyzed using ImageJ software (http://rsb.info.nih. gov/ij/).

\section{Immunoprecipitation}

Cell lysates were pre-cleared with normal mouse or rabbit IgG and protein A sepharose (Amersham Biosciences, GE Healthcare, Waukesha, WI) for $1 \mathrm{~h}$ at $4^{\circ} \mathrm{C}$. The supernatant was then incubated with anti-SUV39h1 or anti-p53 antibodies and protein A sepharose with mixing for $12 \mathrm{~h}$ at $4^{\circ} \mathrm{C}$. The beads were then collected via centrifugation and washed three times with phosphate-buffered saline.

\section{Isolation of nuclear and cytoplasmic extracts}

According to the manufacturer's instruction, cytoplasmic and nuclear extracts were prepared using the NE-PER Nuclear Cytoplasmic Extraction Reagent kit (Pierce, Rockford, IL).

\section{Statistical analysis}

Data were analyzed using one-way analysis of variance in SPSS (IBM, Armonk, NY). The results were considered significant if the $P$ value was $<0.05$. Duncan's multiple range test was performed if the differences between the groups were identi- 
fied as $\alpha=0.05$

\section{ACKNOWLEDGEMENTS}

This research was supported by the Basic Science Research Program through the National Research Foundation of Korea (NRF) funded by the Ministry of Science, ICT and Future Planning (NRF-2015R1A2A2A01004593 and NRF-2019R1A2C100 5219).

\section{CONFLICTS OF INTEREST}

The authors have no conflicting interests.

\section{REFERENCES}

1. Campisi J (2013) Aging, cellular senescence, and cancer. Annu Rev Physiol 75, 685-705

2. Kuilman T, Michaloglou C, Mooi WJ and Peeper DS (2010) The essence of senescence. Genes Dev 24, 2463-2479

3. Dimri GP, Lee X, Basile G et al (1995) A biomarker that identifies senescent human cells in culture and in aging skin in vivo. Proc Natl Acad Sci U S A 92, 9363-9367

4. Zhang R, Chen W and Adams PD (2007) Molecular dissection of formation of senescence-associated heterochromatin foci. Mol Cell Biol 27, 2343-2358

5. Narita M, Nunez S, Heard E et al (2003) Rb-mediated heterochromatin formation and silencing of E2F target genes during cellular senescence. Cell 113, 703-716

6. Kouzarides T (2007) Chromatin modifications and their function. Cell 128, 693-705

7. Ait-Si-Ali S, Guasconi V, Fritsch L et al (2004) A Suv39hdependent mechanism for silencing S-phase genes in differentiating but not in cycling cells. EMBO J 23, 605-615

8. Whetstine JR, Nottke A, Lan F et al (2006) Reversal of histone lysine trimethylation by the JMJD2 family of histone demethylases. Cell 125, 467-481

9. Couture JF, Collazo E, Ortiz-Tello PA et al (2007) Specificity and mechanism of JMJD2A, a trimethyllysine-specific histone demethylase. Nat Struct Mol Biol 14, 689-695

10. Gray SG, Iglesias AH, Lizcano $F$ et al (2005) Functional characterization of JMJD2A, a histone deacetylase- and retinoblastoma-binding protein. J Biol Chem 280, $28507-$ 28518

11. Shi Y, Lan F, Matson C et al (2004) Histone demethylation mediated by the nuclear amine oxidase homolog LSD1. Cell 119, 941-953

12. Wissmann M, Yin N, Muller JM et al (2007) Cooperative demethylation by JMJD2C and LSD1 promotes androgen receptor-dependent gene expression. Nat Cell Biol 9, 347-353
13. Berry WL and Janknecht R (2013) KDM4/JMJD2 histone demethylases: epigenetic regulators in cancer cells. Cancer Res 73, 2936-2942

14. Huang J, Sengupta R, Espejo AB et al (2007) p53 is regulated by the lysine demethylase LSD1. Nature 449, 105-108

15. Johmura $Y$, Sun J, Kitagawa $K$ et al (2016) SCF(Fbxo22)KDM4A targets methylated p53 for degradation and regulates senescence. Nat Commun 7, 10574

16. Wullschleger S, Loewith R and Hall MN (2006) TOR signaling in growth and metabolism. Cell 124, 471-484

17. Morita M, Gravel SP, Hulea L et al (2015) mTOR coordinates protein synthesis, mitochondrial activity and proliferation. Cell Cycle 14, 473-480

18. Ryu SW, Woo JH, Kim YH et al (2006) Downregulation of protein kinase CKII is associated with cellular senescence. FEBS Lett 580, 988-994

19. Kang JY Kim JJ, Jang SY and Bae YS (2009) The p53p21 $1^{\text {ipiNAF1 }}$ pathway is necessary for cellular senescence induced by the inhibition of protein kinase CKII in human colon cancer cells. Mol Cells 28, 489-494

20. Park JH, Kim JJ and Bae YS (2013) Involvement of PI3K-AKTmTOR pathway in protein kinase CKII inhibition-mediated senescence in human colon cancer cells. Biochem Biophys Res Commun 433, 420-425

21. Jang DE, Song J, Park JW, Yoon SH and Bae YS (2020) Protein kinase CK2 activates Nrf2 via autophagic degradation of Keap1 and activation of AMPK in human cancer cells. BMB Rep 53, 272-277

22. Park JH, Lee JH, Park JW et al (2017) Downregulation of protein kinase CK2 activity induces age-related biomarkers in C. elegans. Oncotarget 8, 36950-36963

23. Park JW, Kim JJ and Bae YS (2018) CK2 downregulation induces senescence-associated heterochromatic foci formation through activating SUV39h1 and inactivating G9a. Biochem Biophys Res Commun 505, 67-73

24. Wei J, Ma L, Lai YH et al (2019) Bazedoxifene as a novel GP130 inhibitor for colon cancer therapy. J Exp Clin Cancer Res 38, 63

25. Park JW and Bae YS (2019) Dephosphorylation of p53 Ser392 enhances trimethylation of histone H3 Lys9 via SUV39h1 stabilization in CK2 downregulation-mediated senescence. Mol Cells 42, 773-782

26. Morera L, Lubbert M and Jung M (2016) Targeting histone methyltransferases and demethylases in clinical trials for cancer therapy. Clin Epigenetics 8, 57

27. Monaghan L, Massett ME, Bunschoten RP et al (2019) The emerging role of $\mathrm{H} 3 \mathrm{~K} 9 \mathrm{me} 3$ as a potential therapeutic target in acute myeloid leukemia. Front Oncol 9, 705

28. Fiskus W, Sharma S, Shah B et al (2014) Highly effective combination of LSD1 (KDM1A) antagonist and pan-histone deacetylase inhibitor against human AML cells. Leukemia $28,2155-2164$ 